\title{
SISTEMA INFORMÁTICO PARA EL DISEÑO Y GESTIÓN DE RECORRIDOS VIRTUALES PARA TOURDROID
}

\section{THE COMPUTATIONAL THINKING ABOUT PROMOTING DEVELOPMENT OF SKILLS RELATED CREATIVE SOLVING PROBLEMS}

\author{
Viana de la Cruz Leyva ${ }^{1}$ \\ Yoel Adrián Ortiz Pacheco² \\ Noel Ernesto Enamorado Selema ${ }^{3}$
}

1. Profesora del Departamento de Informática de la Universidad de Granma. Cuba.

vleyva@udg.co.cu

2. Administrador de Redes del Partido Provincial en Granma. Cuba.

3. Profesor de la Universidad de Ciencias Informáticas. Cuba.

\section{Citación sugerida:}

Cruz Leyva, V. de la, Ortiz Pacheco, Y.A. y Enamorado Selema, N.E. (2017). Sistema informático para el diseño y gestión de recorridos virtuales para Tourdroid. 3C TIC: Cuadernos de desarrollo aplicados a las TIC, 6(2), 38-51. DOI: <http://dx.doi.org/10.17993/3ctic.2017.56.38-61/>. 


\section{RESUMEN}

En la Facultad de Ciencias Informáticas de la Universidad de Granma existe el sistema de información geográfica Tourdroid Bayamo para dispositivos móviles con soporte Android en su versión 2.3 o superior. Es una guía virtual que asocia información geográfica y visual de sitios de interés histórico, económico y cultural de la región. La investigación tiene como objetivo crear un sistema informático para agilizar el proceso de diseño y gestión de los recorridos virtuales para Tourdroid.

\section{ABSTRACT}

In the Faculty of Computer Science of the University of Granma there is the geographical information system Tourdroid Bayamo for mobile devices with Android support in version 2.3 or higher. It is a virtual guide that associates geographic and visual information of sites of historical, economic and cultural interest of the region. The research aims to create a computer system to streamline the process of design and management of virtual tours for Tourdroid.

\section{PALABRAS CLAVE}

Android, móviles, Tourdroid, virtual.

\section{KEY WORDS}

Android, mobile, Tourdroid, virtual. 


\section{INTRODUCCIÓN}

El desarrollo social y tecnológico de una región está muy ligado a la gestión y potencialización de su territorio. Para realizar la gestión de un territorio es necesario definir la localización y características esenciales del mismo, de manera que exista claridad para generar información segura que permita optimizar la planificación y gestión de las actividades económicas. Los SIG son las herramientas que se han desarrollado mundialmente para llevar a cabo estas tareas.

Un SIG es una colección organizada de hardware, software, datos geográficos y personal designado para capturar, almacenar, actualizar, manipular, analizar y mostrar todas las formas de información geográfica referenciada, o sea, un sistema capaz de mantener y usar datos que están asociados a lugares en la superficie terrestre.

En la actualidad, los SIG tienen una fuerte implantación en los llamados servicios basados en la localización (LBS, Location Based Service) debido a la masificación de los sistemas de posicionamiento global (GPS, Global Position System) integrado en dispositivos móviles. Los LBS permiten a los dispositivos móviles con GPS mostrar su ubicación respecto a puntos de interés fijos: restaurantes, gasolineras, cajeros, hoteles, entre otros.

Los SIG, con la evolución de la telefonía móvil, han encontrado una plataforma de interés para su implementación. En el mundo se han realizado varios SIG para celulares, ejemplo de esto son, MapDroid y Locus.

En Cuba, la empresa Geosi desarrolló el SIG Andariego para dispositivos móviles con sistema operativo Android. Esta herramienta brinda información detallada sobre todas las provincias de Cuba, además cuenta con servicio GPS que le permite al usuario ver su localización en el mapa y su recorrido mientras se mueve. Para su actualización, es necesario acceder a Internet a través de una computadora personal y descargar los datos actualizados.

En la Facultad de Ciencias Informáticas de la Universidad de Granma se desarrolló el SIG Tourdroid Bayamo para dispositivos móviles con soporte Android en su versión 2.3 o superior. Tourdroid es una guía virtual que asocia información geográfica y visual de sitios de interés histórico, económico y cultural de una región. La información que brinda a los usuarios solo depende de la almacenada en la base de datos, por lo que sería necesario enfrentarse en una nueva versión de la aplicación, para agregarle nuevas funcionalidades.

Define una arquitectura estrictamente regida por la información almacenada en la base de datos, pensada inicialmente para hacerlo adaptable a cambios en tiempo real y que lograra independencia del cliente con el equipo de desarrollo. Hoy, la información que se brinda se considera obsoleta debido a que esta no puede ser actualizada una vez desplegado el producto, a menos que se sobrescriba la base de datos existente en los terminales móviles.

La obsolescencia de los datos almacenados en Tourdroid influye de forma negativa en el nivel de aceptación de la aplicación, ya que las instituciones promovidas podrían sufrir cambios en su estructura interna y no existe un mecanismo para la actualización de su información. Solamente depende de la capacidad de respuesta de un equipo de desarrollo que no es 


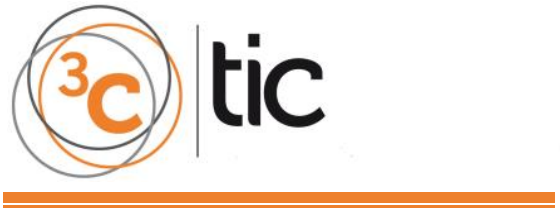

factible para los usuarios ni la empresa que la comercialice pues contradice varios principios de los sistemas de información geográfica, influyendo negativamente para definirlo como un producto de calidad.

Analizada la problemática antes expuesta se plantea como problema a resolver: ¿Cómo mejorar el diseño y gestión de la información contenida en la base de datos de Tourdroid?

El objeto de estudio de la presente investigación se enmarca en el diseño y gestión de recorridos virtuales.

Una vez analizado el problema científico en cuestión, se define como objetivo general de la investigación: desarrollar el sistema informático para el diseño y gestión de recorridos virtuales para Tourdroid.

El campo de acción se centra en el diseño y gestión de recorridos virtuales para Tourdroid.

Se establece como idea a defender que con el desarrollo del sistema informático para el diseño y gestión de recorridos virtuales para Tourdroid se agilizará el proceso de actualización de la información almacenada en su base de datos.

\section{SISTEMA DE INFORMACIÓN GEOGRÁFICA TOURDROID}

Tourdroid es un sistema de información geográfica para dispositivos móviles con sistema operativo Android en su versión 2.3 o superior, capaz de brindar información geográfica y visual de los distintos sitios de interés de un entorno. Aunque inicialmente fue concebido para la ciudad de Bayamo, la herramienta posee una arquitectura adaptable para estandarizarla a otros entornos, lo cual se sustenta en que solo es necesario la modificación de los datos contenidos en su base de datos a menos que se incremente un valor agregado a sus funcionalidades.

El sistema permite:

$\checkmark$ Visualizar la información descriptiva del recorrido virtual perteneciente al lugar escogido.

$\checkmark$ Observar las imágenes panorámicas cilíndricas referentes a las salas de un recorrido virtual.

$\checkmark$ Visualizar la descripción correspondiente a la sala, lo que le facilita a los usuarios conocer en detalle la instalación.

$\checkmark$ Ampliar y disminuir un área de la imagen panorámica perteneciente a la sala visualizada. Con ello, se consigue mayor exactitud en la visualización de los objetos pequeños que se encuentran en la escena. 
$\checkmark$ Desplazarse en una imagen panorámica de forma horizontal y vertical, brindándole a los usuarios mayor efecto de realismo.

$\checkmark$ Seleccionar un objeto de interés incluido en una sala.

$\checkmark$ Apreciar la descripción correspondiente al objeto seleccionado, lo que le facilita a los usuarios conocer información detallada de los elementos que forman parte de la escena.

$\checkmark$ Visualizar una imagen ampliada del objeto seleccionado correspondiente a una sala de un recorrido virtual.

La herramienta posee un mecanismo de acceso a los datos, que recibe las solicitudes de almacenamiento o recuperación de información y envía las respuestas a las peticiones, este se basa en la correcta y precisa manipulación de los datos.

\subsection{RECORRIDOS VIRTUALES EN TOURDROID}

La visualización de los recorridos virtuales en Tourdroid se realiza de la forma tradicional, es decir, basado en la visualización en $360^{\circ}$ de un conjunto de imágenes panorámicas relacionadas entre sí. No obstante, están implementadas para dispositivos móviles con sistema operativo Android, el cual cuenta con la librería de código abierto PanoramaGLAndroid que posee la propiedad de visualizar imágenes panorámicas esféricas, cilíndricas y cúbicas, y que además permite ampliar y disminuir la imagen visualizada mediante el zoom. También facilita el desplazamiento en la panorámica hacia cualquier dirección, con el uso del acelerómetro del teléfono inteligente. Ofrece compatibilidad con OpenGL ES 1.1 y soporte para el uso de hotspots. En contraposición, para su correcto funcionamiento se necesita la máquina virtual de Java para Android, Dalvik Virtual Machine, instalado en su dispositivo móvil.

\section{PROPUESTA DE SOLUCIÓN}

Para lograr el desarrollo de una aplicación con calidad que satisfaga las necesidades del cliente, en el trabajo actual se define la metodología que guiará el proceso de desarrollo del software, así como los lenguajes y herramientas que se utilizarán para el desarrollo de la aplicación. Se describen las funcionalidades que debe brindar el sistema informático propuesto, así como su arquitectura y modelo de diseño. 


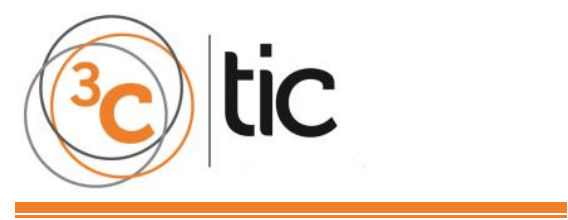

\subsection{ESTUDIOS DE SIMILARES}

\section{DVista Virtual Tour Standard}

3DVista Virtual Tour Standard permite crear visitas virtuales espectaculares que pueden ser incluidas en cualquier web y vistas desde cualquier dispositivo u ordenador. Convierte un grupo de fotos en una escena de 360․ Incluye 3DVista Stitcher 4 y un Publicador que convierte los panoramas en visitas virtuales de alta calidad con interfaz gráfico totalmente personalizable (“3DVista," 2016).

\section{DVista Virtual Tour Pro}

3DVista Virtual Tour Pro permite crear visitas virtuales multimedia que cautivan por su interactividad e impresionante rango de opciones, tales como Live Panorama, HDR Adaptativo, Hotspots, Piloto automático, Video interactivo, Albums de Fotos, Planos y Audio Inmersivo. Incluye también 3DVista Stitcher 4 y un Publicador. 3DVista ha desarrollado la tecnología "HDR Adaptativo" que adapta la luz de un panorama según la zona donde mire el usuario. Con solo unos clics se obtiene visitas virtuales profesionales compatibles con todos los dispositivos móviles, tabletas y ordenadores ("3DVista," 2016).

\section{Dermandar}

Dermandar es una herramienta online que permite hacer fotografías panorámicas de forma muy cómoda e insertarlas en los sitios web. Tiene versiones para Android y iPhone (Google Play - Apple Store) que facilitan el trabajo, pues son las que hacen el montado de las fotografías. Soporta HTML5 (Raúl, 2012).

\subsection{DESCRIPCIÓN DE LA SOLUCIÓN PROPUESTA}

Se propone el desarrollo de un sistema informático que posibilite el diseño y gestión de recorridos virtuales para Tourdroid, mediante un ambiente amigable. El sistema debe permitir la gestión de la información almacenada, la creación, modificación y eliminación de recorridos virtuales, salas y hotspots. Los datos geográficos referentes a los recorridos virtuales podrán ser verificados mediante su visualización en un espacio geográfico simulado por un mapa. Posibilitará generar una base de datos con la información del sistema para su posterior divulgación. Se propone además el desarrollo de un sistema de transmisión de datos para la actualización de la información almacenada en los dispositivos móviles que cuenten con Tourdroid. 


\subsection{METOdOLOGÍA DE DESARROLlO DE SOFTWARE ESCOGIDA}

Las metodologías de desarrollo de software brindan un conjunto de procedimientos, técnicas, herramientas y soporte documental que ayuda a los desarrolladores a realizar un mejor producto, al estructurar, planificar y controlar el proceso de desarrollo del software.

Una metodología es un conjunto de métodos eficientes orientados a conseguir un objetivo definido, un grupo de procesos que, organizados, brindan una secuencia de pasos a seguir para obtener los hitos propuestos y, finalmente el producto (2010).

Las metodologías de desarrollo de software se pueden clasificar en ágiles y robustas, y la aplicación de una de ellas depende de las características del producto que se desee desarrollar. En la presente investigación se utilizará un enfoque de desarrollo ágil debido a las características del software que se realizará.

Se seleccionó como metodología de desarrollo de software SXP, porque al ser un híbrido creado a partir de lo mejor de SCRUM y XP, se adapta al proyecto en cuestión y permite cambios en los requisitos en dependencia de las necesidades del usuario. El equipo de desarrollo está compuesto por pocas personas y esta metodología es ideal para equipos pequeños. Solo genera los artefactos necesarios, lo que permite utilizar el tiempo en otras actividades y agilizar el desarrollo del software. Además permite mantener de forma organizada los artefactos que se generan por cada una de las fases.

SXP

Es un híbrido entre las metodologías SCRUM y XP, la cual ofrece una estrategia tecnológica a partir de la introducción de procedimientos ágiles que permiten actualizar los procesos de software para el mejoramiento de la actividad productiva. Se fomenta el desarrollo de la creatividad, se aumenta el nivel de preocupación y responsabilidad de los miembros del equipo y se ayuda al líder del proyecto a tener un mejor control del mismo. Consiste en una programación rápida o extrema, cuya particularidad es tener como parte del equipo, al usuario final, pues es uno de los requisitos para llegar al éxito del proyecto (Abel Meneses, 2009).

SXP cuenta con cuatro fases:

1. Planificación-definición: en esta fase se establece la visión, se fijan las expectativas y se realiza el aseguramiento del financiamiento del proyecto.

2. Desarrollo: aquí es donde se realiza la implementación del sistema hasta que se encuentre listo para ser entregado, además se realizan las pruebas al software.

3. Entrega: puesta en marcha del producto.

4. Mantenimiento: donde se realiza el soporte de la aplicación para el cliente. 


\subsection{LENGUAJES Y HERRAMIENTAS}

Python ha sido diseñado por Guido Van Rossum y está en un proceso de continuo desarrollo por una gran comunidad de desarrolladores. Es un lenguaje de programación interpretado y orientado a objetos. Está libremente disponible en archivos binarios o de código fuente. Una ventaja fundamental de Python es la gratuidad de su intérprete.

Python presenta una serie de ventajas que lo hacen muy atractivo, tanto para su uso profesional como para el aprendizaje de la programación. Entre las más interesantes desde el punto de vista didáctico aparecen las siguientes (Isabel García, 2003):

$\checkmark$ Es un lenguaje muy expresivo, es decir, los programas Python son muy compactos: un programa Python suele ser más corto que su equivalente en lenguajes como $C$.

$\checkmark$ Es muy legible. Su sintaxis es muy elegante y permite la escritura de programas cuya lectura resulta más fácil que si se utilizaran otros lenguajes de programación.

$\checkmark$ Ofrece un entorno interactivo que facilita la realización de pruebas.

$\checkmark$ Puede usarse como lenguaje imperativo procedimental o como lenguaje orientado a objetos.

$\checkmark$ Posee un rico juego de estructuras de datos que se pueden manipular de modo sencillo.

$\checkmark$ Es fácil de comprender y está considerado por muchos que será el lenguaje de programación de elección por los nuevos programadores.

Se selecciona del lenguaje de programación Python para el desarrollo de la aplicación porque es un lenguaje libre, multiplataforma, orientado a objetos, muy fácil de aprender y tiene una sintaxis muy simple que hace que los programas escritos en él sean legibles.

\section{Lenguaje unificado de modelado}

UML (Unified Modeling Language) es el estándar industrial de notación de modelado para sistemas orientados a objetos, constituye la plataforma inicial para el desarrollo rápido de aplicaciones. Es un lenguaje gráfico para visualizar, especificar, construir y documentar artefactos de un software (Roger S. Pressman, 2002). Se utiliza UML para modelar el sistema, ya que se compone de elementos que permiten la representación del software mediante diagramas. Además, sirve de guía a la mayoría de los procesos orientados a objetos, capta la información sobre la estructura estática y el comportamiento dinámico del sistema.

\section{Herramienta CASE}

De acuerdo a Roger S. Pressman, las herramientas CASE (Computer Aided Software Engineering) "Son un complemento de la caja de herramientas del ingeniero del software que le proporciona, la posibilidad de automatizar actividades manuales y de mejorar su visión 
general de la ingeniería. Al igual que las herramientas de ingeniería y diseño asistidos por computadora que utilizan de otras disciplinas, las herramientas CASE ayudan a asegurar que la calidad sea algo diseñado antes de llegar a construir el producto" (Roger S. Pressman, 2002).

Estas herramientas ayudan a los gestores y practicantes de la ingeniería del software en todas las actividades asociadas a los procesos de software. Automatizan las actividades de gestión de proyectos, gestionan todos los productos de los trabajos elaborados a través del proceso y ayudan a los ingenieros en el trabajo de análisis, diseño y codificación. Las herramientas CASE se pueden integrar dentro de un entorno sofisticado.

\section{Visual Paradigm}

Visual Paradigm es una herramienta profesional que soporta el ciclo de vida del desarrollo de software. Ayuda a una rápida construcción de aplicaciones de calidad a un menor coste. Permite dibujar todos los tipos de diagramas de clases, generar código desde diagramas y documentación. Es compatible con sistemas GNU/Linux y está registrado bajo la licencia de software libre.

Algunas de las principales ventajas que ofrece son las siguientes (n.d.):

$\checkmark$ Usa un lenguaje estándar común a todo el equipo de desarrollo y facilita la comunicación.

$\checkmark$ Tiene capacidades de ingeniería directa e inversa.

Tiene modelos y códigos que permanecen sincronizados en todo el ciclo de desarrollo.

$\checkmark$ Presenta disponibilidad de múltiples versiones, para cada necesidad y múltiples plataformas.

Se emplea Visual Paradigm por sus ventajas y facilidad de uso, además, utiliza UML como notación para elaborar los modelos y comunicar de manera eficiente a todos los agentes del proyecto aquellas decisiones que se toman con respecto a la arquitectura del sistema en discusión. La misma permite realizar rápidamente el modelado necesario para el desarrollo de la aplicación.

\section{Entorno de desarrollo integrado}

Un entorno de desarrollo integrado (IDE, Integrated Development Environment) es un conjunto de herramientas de programación para escribir aplicaciones, todo activado desde unos menús e interfaces de usuario común. Es un procedimiento estándar necesario para el desarrollo de programas (n.d.).

Estos incluyen un editor de código fuente, un compilador y usualmente un depurador que trabajan conjuntamente al construir un software. El IDE le sigue la pista a todos los archivos 
relacionados con un proyecto y provee una interfaz central para el código fuente que escribe. A continuación se describe el empleado en el desarrollo de la aplicación.

Geany es un editor de texto con las características básicas de un entorno de desarrollo integrado, desarrollado para que tuviera pocas dependencias de otros paquetes. Su principal objetivo es ser lo más independiente posible de entornos de escritorios especiales, solo requiere de las bibliotecas en tiempo de ejecución GTK2. Posee características como: autocompletado en la construcción del código fuente, coloreado de sintaxis, compatible con la mayoría de los lenguajes de programación, verifica que todas las etiquetas estén cerradas y consume poca memoria RAM (Random Access Memory). Se puede ejecutar bajo varias plataformas incluidas Windows y Linux, su código está disponible bajo los términos de la Licencia Pública General de GNU (n.d.).

\subsection{ESPECIFICACIÓN DE LOS REQUISITOS DEL SISTEMA}

Es vital en la producción de un software lograr una comunicación efectiva entre los usuarios y el equipo de desarrollo, con el objetivo de llegar a un entendimiento de lo que se debe hacer. La correcta identificación de los requisitos es el punto de partida para desarrollar un producto de calidad que satisfaga para el cliente.

"Un requerimiento es una característica que el sistema debe tener o es una restricción que el sistema debe satisfacer para ser aceptada por el cliente" (n.d.).

Tras conocer las necesidades del cliente se identificaron los requisitos funcionales (RF) y no funcionales (RNF).

\section{Requisitos funcionales}

Los requisitos funcionales son declaraciones de las características que debe proporcionar el sistema. Describen la interacción entre el sistema y su ambiente independientemente de su implementación. El ambiente incluye al usuario y cualquier otro sistema externo que interactúa con el sistema (n.d.).

A continuación se muestran los RF de la aplicación a desarrollar:

1. Autenticar usuario: el sistema debe validar una vez que el usuario intente acceder al sistema que el nombre de usuario y contraseña sean correctos.

2. Gestionar usuario: permite al administrador del sistema, insertar, modificar o eliminar un usuario.

3. Gestionar un recorrido virtual: permite al usuario insertar, modificar o eliminar un recorrido virtual. 
4. Gestionar las salas o espacios incluidos en el recorrido virtual: permite al usuario insertar, modificar o eliminar las imágenes panorámicas, así como los vínculos entre ellas.

5. Gestionar objetos: permite al usuario insertar, modificar o eliminar los objetos más importantes de una sala.

6. Insertar mapa: permite al usuario agregar un mapa del entorno que mostrará la información geográfica de los recorridos virtuales.

7. Generar actualización: permite al usuario generar la base de datos de Tourdroid incluyendo los últimos cambios realizados.

8. Actualizar Tourdroid: permite al usuario actualizar la base de datos de Tourdroid en los dispositivos móviles que cuenten con dicha aplicación.

\section{Requisitos no funcionales}

Los requisitos no funcionales son aquellos requerimientos que no se refieren directamente a las funciones específicas que proporciona el sistema, sino a las propiedades emergentes de este como la fiabilidad, capacidad de almacenamiento y el tiempo de respuesta. De forma alternativa, definen las restricciones del sistema como la capacidad de los dispositivos de entrada/salida y las representaciones de datos que se utilizan en las interfaces del sistema (n.d.).

A continuación, se muestran los requisitos no funcionales del sistema:

- Requisitos de usabilidad: El sistema propuesto podrá ser usado por usuarios con conocimiento básicos de informática, ya que estará estructurado de forma sencilla. El sistema proporcionará un mejor escenario en el proceso de diseñar y gestionar los recorridos virtuales para Tourdroid.

- Requisitos de seguridad: El sistema deberá garantizar el tratamiento adecuado de las excepciones mediante el lanzamiento de un tipo de excepción en dependencia de la situación anómala que ocurra, manejándola, una vez que se conozca el error ocurrido. El sistema deberá garantizar que el usuario reciba de forma segura la actualización de la información de la base de datos de Tourdroid. El sistema deberá garantizar que para que un usuario pueda acceder al sistema, antes, debe estar autenticado con un usuario válido. El sistema solo le mostrará a cada usuario las funcionalidades sobre las cuales tiene permiso.

- Requisitos de software: Sistemas operativos: GNU/Linux o Microsoft Windows.

- Requisitos de hardware: El sistema deberá desplegarse en una PC, que cuente con los requerimientos mínimos de un microprocesador Pentium IV de $3.0 \mathrm{GHz}$, con una memoria RAM de $512 \mathrm{MB}$ y un disco duro con al menos $50 \mathrm{MB}$ de capacidad disponible. Los dispositivos móviles que cuenten con Tourdroid, necesitan tener 


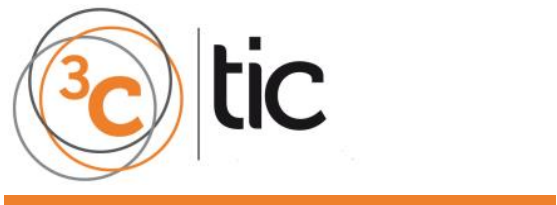

implementado el estándar IEEE 802.11. En la red donde se despliegue el producto se debe disponer de un punto de acceso inalámbrico.

- Restricciones de diseño e implementación: Uso de plataforma libre, se programará en el lenguaje Python, se hará uso del Qt Designer para el diseño de las interfaces de la aplicación y se utilizará el comando de Python pyuic4 para convertir las interfaces.ui creadas en el Qt Designer a interfaces.py.

\subsection{DESCRIPCIÓN DE LAS HISTORIAS DE USUARIO}

A continuación se describe la historia de usuario (HU) Autenticar_usuario de la aplicación a desarrollar.

Tabla 2. Descripción de la HU Autenticar_Usuario.

\begin{tabular}{|l|l|}
\hline Número: 2 & Nombre historia de usuario: Autenticar_usuario \\
\hline $\begin{array}{l}\text { Usuario: Yoel Adrian Ortiz Pacheco } \\
\text { Noel Ernesto Enamorado Selema }\end{array}$ & Iteración asignada: 1 \\
\hline & \multicolumn{2}{|c|}{ Puntos estimados: 1} \\
\hline Programador responsable: Yoel Adrian Ortiz Pacheco \\
\hline Prioridad en negocio: Alta & $\begin{array}{l}\text { Riesgo en desarrollo: Alto } \\
\text { Descripción: la HU comienza cuando el usuario desea acceder a la aplicación. Para autenticarse se } \\
\text { debe insertar el nombre de usuario y la contraseña correctamente. Al presionar el botón “Aceptar” } \\
\text { terminará la HU. }\end{array}$ \\
\hline \begin{tabular}{l} 
Prototipo de interfaz: Figura B.1 \\
\hline
\end{tabular}
\end{tabular}

Fuente: elaboración propia.

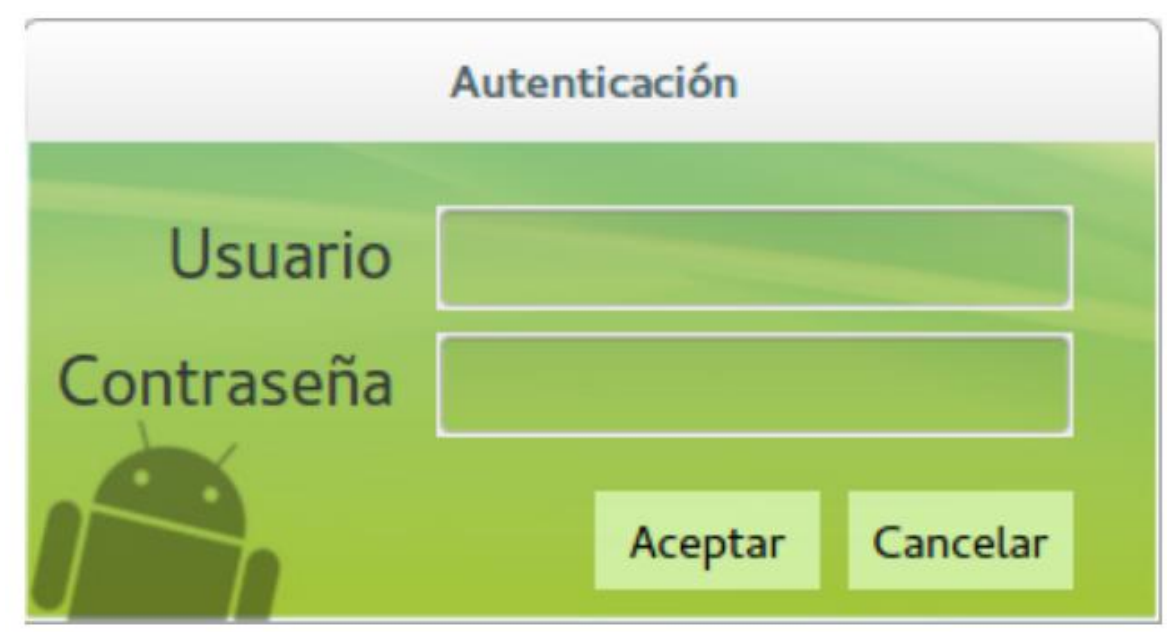

Ilustración 1. Autenticar_Usuario. 


\section{CONCLUSIONES}

Con el desarrollo del sistema informático para el diseño y gestión de recorridos virtuales para Tourdroid, se obtuvo un sistema que agiliza el proceso de actualización de su información, dándole solución a la problemática existente. La información que brindará Tourdroid podría considerarse actualizada, lo que aumentaría el nivel de aceptación de la aplicación. El desarrollo del producto estuvo guiado por la metodología de desarrollo de software SXP, la cual permitió hacer uso de las ventajas del manifiesto ágil y documentar correctamente toda la información manejada, mediante un expediente de proyecto con artefactos bien definidos.

Un estudio de sistemas similares reveló que estos no se adaptaban a los requerimientos de la aplicación a implementar, ya que sus recorridos virtuales definen una arquitectura distinta a los de Tourdroid y se desarrollan sobre tecnologías privativas; aunque apoyó en la concepción del negocio para el desarrollo del sistema.

El sistema ofrecido permite, mediante un ambiente amigable, la gestión de la información almacenada en la base de datos de Tourdroid, y brinda un sistema de transmisión inalámbrica para su actualización. Implementándose correctamente todos los requisitos que el cliente solicitó, con la adecuada aplicación de metodologías, herramientas y lenguajes de código abierto.

El servicio web como mecanismo de comunicación, unido a la tecnología de transmisión inalámbrica IEEE 802.11, constituyeron una solución correcta para lograr la transferencia de los datos actualizados hacia los usuarios finales. Se consiguió mediante la implementación de una nueva funcionalidad a Tourdroid, como un valor agregado, algo que solo era posible al modificar manualmente la base de datos contenida en el dispositivo móvil.

Se validó el sistema mediante una estrategia de pruebas que demostró el correcto funcionamiento de la aplicación, así como la satisfacción del cliente con el producto ofrecido. Las pruebas realizadas fueron un elemento crítico para la garantía de calidad del software y representaron una revisión final de las especificaciones, el diseño y la codificación. 


\section{REFERENCIAS BIBLIOGRÁFICAS}

3DVista. (2016). Retrieved June 13, 2017, from http://www.3dvista.com/es/products/virtualtour

Abel Meneses. (2009). SXP, Metodología ágil para el desarrollo de software.

Geany. (n.d.). Retrieved from http://geany.org

Integrated development environment. (n.d.). Retrieved from http://www.pcmag.com/ encyclopedia/term/44707/ide.

Isabel Garcia, A. M. (2003, September). Introducción a la programación con Python.

Juan Pablo Quiroga. (n.d.). Requerimientos Funcionales y No Funcionales.

Metodologías Tradicionales vs. Metodologías Agiles. (2010). Universidad Técnica Particular de Loja, Ecuador.

Raúl. (2012, November 21). En la nube TIC. Retrieved June 13, 2017, from http://www.enlanubetic.com.es/2012/11/imagenes-panoramicas-condermandar.html

Roger S. Pressman. (2002). Ingeniería del Software: Un enfoque práctico (Quinta). McGrawHill.

VISUAL PARADIGM. UML tool, business process modeler and database desig-ner for software development team. (n.d.). Retrieved from http://www.visual-paradigm.com 